\title{
Contrasting Behavior of Bis[(2-(4,4-dimethyl-2-
}

\section{oxazolinyl)phenyl] Chalcogenides (Se/Te) toward}

\section{Mercuric Chloride: Facile Cleavage of Te-C Bond}

\author{
Sandeep D. Apte ${ }^{a}$, Sanjio S. Zade ${ }^{a}$, Harkesh B. Singh ${ }^{a}$, and Ray J. Butcher ${ }^{b}$ \\ a Department of Chemistry, Indian Institute of Technology Bombay, Powai, Mumbai 400076, \\ India.
}

${ }^{\mathrm{b}}$ Department of Chemistry, Howard University, Washington, D.C. 20059, USA

Email: chhbsia@chem.iitb.ac.in

SUPPORTING INFORMATION 
Table 1. Crystal data and structure refinement for $\mathbf{5}$

Identification code hgoxse

Empirical formula

$\mathrm{C} 22 \mathrm{H} 24 \mathrm{Cl} 2 \mathrm{Hg}$ N2 O2 Se

Formula weight

698.88

Temperature

293(2) K

Wavelength

$0.71073 \AA$

Crystal system

Monoclinic

Space group

$\mathrm{P} 2(1) / \mathrm{c}$

Unit cell dimensions

$\mathrm{a}=12.589(2) \AA$ $\square=90^{\circ}$.

$\mathrm{b}=10.5362(19) \AA$ $\square=106.010(12)^{\circ}$.

$c=18.866(3) \AA$ $\square=90^{\circ}$.

Volume

2405.3(7) $\AA^{3}$

Z

4

Density (calculated)

$1.930 \mathrm{Mg} / \mathrm{m}^{3}$

Absorption coefficient

$8.155 \mathrm{~mm}^{-1}$

$\mathrm{F}(000)$

1336

Crystal size

$0.72 \times 0.63 \times 0.56 \mathrm{~mm}^{3}$

Theta range for data collection

2.24 to $27.50^{\circ}$.

Index ranges

$0<=\mathrm{h}<=16,0<=\mathrm{k}<=13,-24<=\mathrm{l}<=23$

Reflections collected

5693

Independent reflections

$5452[\mathrm{R}(\mathrm{int})=0.0252]$

Completeness to theta $=27.50^{\circ}$

$98.6 \%$

Absorption correction

SHELXA 
Max. and min. transmission

Refinement method

Data / restraints / parameters

Goodness-of-fit on $\mathrm{F}^{2}$

Final $\mathrm{R}$ indices [I $>2 \operatorname{sigma}(\mathrm{I})]$

$\mathrm{R}$ indices (all data)

Extinction coefficient

Largest diff. peak and hole
0.7252 and 0.2766

Full-matrix least-squares on $\mathrm{F}^{2}$

5452 / 0 / 300

1.042

$\mathrm{R} 1=0.0373, w \mathrm{R} 2=0.0766$

$\mathrm{R} 1=0.0615, \mathrm{wR} 2=0.0851$

$0.00184(12)$

1.085 and -0.656 e. $\AA^{-3}$

Table 2. Atomic coordinates ( $\left.\mathrm{x} 10^{4}\right)$ and equivalent isotropic displacement parameters $\left(\AA^{2} \mathrm{x}\right.$ $10^{3}$ ) for 5 . U(eq) is defined as one third of the trace of the orthogonalized $U^{i j}$ tensor.

\begin{tabular}{lcccc}
\hline & & & & \\
& & & & \\
& & & & \\
& & & & \\
$\mathrm{Hg}$ & $7935(1)$ & $1253(1)$ & $2233(1)$ & $47(1)$ \\
$\mathrm{Se}$ & $6416(1)$ & $-88(1)$ & $1169(1)$ & $36(1)$ \\
$\mathrm{Cl}(1)$ & $8280(2)$ & $163(2)$ & $3388(1)$ & $70(1)$ \\
$\mathrm{Cl}(2)$ & $7783(2)$ & $3518(2)$ & $2283(1)$ & $76(1)$ \\
$\mathrm{O}(1)$ & $9979(3)$ & $-333(4)$ & $816(2)$ & $51(1)$ \\
$\mathrm{O}(2)$ & $5072(4)$ & $3880(4)$ & $784(2)$ & $53(1)$ \\
$\mathrm{N}(1)$ & $9081(3)$ & $705(4)$ & $1505(2)$ & $34(1)$ \\
$\mathrm{N}(2)$ & $5178(4)$ & $1961(4)$ & $1320(3)$ & $42(1)$ \\
$\mathrm{C}(11)$ & $7346(5)$ & $-1476(5)$ & $1026(3)$ & $36(1)$
\end{tabular}




\begin{tabular}{|c|c|c|c|c|}
\hline$C(12)$ & $6808(5)$ & $-2629(6)$ & $947(3)$ & $50(2)$ \\
\hline $\mathrm{C}(13)$ & $7338(7)$ & $-3745(6)$ & $861(4)$ & $62(2)$ \\
\hline$C(14)$ & $8424(7)$ & $-3712(6)$ & $848(4)$ & $63(2)$ \\
\hline$C(15)$ & $8982(6)$ & $-2570(6)$ & $927(4)$ & $51(2)$ \\
\hline$C(16)$ & $8475(5)$ & $-1440(5)$ & $1033(3)$ & $37(1)$ \\
\hline $\mathrm{C}(17)$ & $9146(4)$ & $-277(5)$ & $1146(3)$ & $37(1)$ \\
\hline $\mathrm{C}(18)$ & $10669(6)$ & $766(7)$ & $1095(4)$ & $56(2)$ \\
\hline C(19) & $9968(4)$ & $1617(6)$ & $1428(3)$ & $40(1)$ \\
\hline$C(110)$ & $9441(6)$ & $2677(6)$ & $923(4)$ & $63(2)$ \\
\hline $\mathrm{C}(111)$ & $10602(6)$ & $2112(7)$ & $2176(4)$ & $59(2)$ \\
\hline $\mathrm{C}(21)$ & $6491(4)$ & $1023(5)$ & $369(3)$ & $33(1)$ \\
\hline $\mathrm{C}(22)$ & $6991(5)$ & $644(6)$ & $-160(3)$ & $43(1)$ \\
\hline $\mathrm{C}(23)$ & $7090(5)$ & $1484(6)$ & $-709(3)$ & $48(2)$ \\
\hline $\mathrm{C}(24)$ & $6726(5)$ & $2707(6)$ & $-715(3)$ & $46(1)$ \\
\hline$C(25)$ & $6191(5)$ & $3090(5)$ & $-205(3)$ & $41(1)$ \\
\hline$C(26)$ & $6055(4)$ & $2266(5)$ & $336(3)$ & $34(1)$ \\
\hline $\mathrm{C}(27)$ & $5431(4)$ & $2667(5)$ & $851(3)$ & $37(1)$ \\
\hline $\mathrm{C}(28)$ & $4522(8)$ & $4027(7)$ & $1362(5)$ & $69(2)$ \\
\hline C(29) & $4457(5)$ & $2716(6)$ & $1667(3)$ & $49(2)$ \\
\hline$C(210)$ & $3328(7)$ & $2154(10)$ & $1433(8)$ & $103(4)$ \\
\hline $\mathrm{C}(211)$ & $4912(10)$ & $2669(10)$ & $2492(5)$ & $93(3)$ \\
\hline
\end{tabular}


Table 3. Bond lengths $[\AA]$ and angles $\left[{ }^{\circ}\right]$ for 5 .

$\begin{array}{ll}\mathrm{Hg}-\mathrm{N}(1) & 2.322(4) \\ \mathrm{Hg}-\mathrm{Cl}(1) & 2.3945(17) \\ \mathrm{Hg}-\mathrm{Cl}(2) & 2.3984(18) \\ \mathrm{Hg}-\mathrm{Se} & 2.7501(7) \\ \mathrm{Se}-\mathrm{C}(21) & 1.933(5) \\ \mathrm{Se}-\mathrm{C}(11) & 1.940(5) \\ \mathrm{O}(1)-\mathrm{C}(17) & 1.359(6) \\ \mathrm{O}(1)-\mathrm{C}(18) & 1.457(7) \\ \mathrm{O}(2)-\mathrm{C}(27) & 1.350(6) \\ \mathrm{O}(2)-\mathrm{C}(28) & 1.451(7) \\ \mathrm{N}(1)-\mathrm{C}(17) & 1.253(7) \\ \mathrm{N}(1)-\mathrm{C}(19) & 1.510(7) \\ \mathrm{N}(2)-\mathrm{C}(27) & 1.262(7) \\ \mathrm{N}(2)-\mathrm{C}(29) & 1.488(7) \\ \mathrm{C}(11)-\mathrm{C}(12) & 1.379(7) \\ \mathrm{C}(11)-\mathrm{C}(16) & 1.418(8) \\ \mathrm{C}(12)-\mathrm{C}(13) & 1.383(9) \\ \mathrm{C}(13)-\mathrm{C}(14) & 1.375(11) \\ \mathrm{C}(14)-\mathrm{C}(15) & 1.380(9) \\ \mathrm{C}(15)-\mathrm{C}(16) & \\ \mathrm{C}(16)-\mathrm{C}(17) & \end{array}$




\begin{tabular}{|c|c|}
\hline $\mathrm{C}(18)-\mathrm{C}(19)$ & $1.511(9)$ \\
\hline C(19)-C(110) & $1.499(9)$ \\
\hline C(19)-C(111) & $1.511(8)$ \\
\hline$C(21)-C(22)$ & $1.377(7)$ \\
\hline $\mathrm{C}(21)-\mathrm{C}(26)$ & $1.415(7)$ \\
\hline$C(22)-C(23)$ & $1.393(8)$ \\
\hline $\mathrm{C}(23)-\mathrm{C}(24)$ & $1.367(8)$ \\
\hline$C(24)-C(25)$ & $1.377(8)$ \\
\hline $\mathrm{C}(25)-\mathrm{C}(26)$ & $1.387(7)$ \\
\hline$C(26)-C(27)$ & $1.468(7)$ \\
\hline $\mathrm{C}(28)-\mathrm{C}(29)$ & $1.508(9)$ \\
\hline C(29)-C(210) & $1.489(10)$ \\
\hline$C(29)-C(211)$ & $1.505(10)$ \\
\hline $\mathrm{N}(1)-\mathrm{Hg}-\mathrm{Cl}(1)$ & $115.64(12)$ \\
\hline $\mathrm{N}(1)-\mathrm{Hg}-\mathrm{Cl}(2)$ & $109.82(12)$ \\
\hline $\mathrm{Cl}(1)-\mathrm{Hg}-\mathrm{Cl}(2)$ & $116.00(7)$ \\
\hline $\mathrm{N}(1)-\mathrm{Hg}-\mathrm{Se}$ & $82.03(10)$ \\
\hline $\mathrm{Cl}(1)-\mathrm{Hg}-\mathrm{Se}$ & $109.46(6)$ \\
\hline $\mathrm{Cl}(2)-\mathrm{Hg}-\mathrm{Se}$ & $119.51(5)$ \\
\hline $\mathrm{C}(21)-\mathrm{Se}-\mathrm{C}(11)$ & $100.7(2)$ \\
\hline $\mathrm{C}(21)-\mathrm{Se}-\mathrm{Hg}$ & $94.88(15)$ \\
\hline $\mathrm{C}(11)-\mathrm{Se}-\mathrm{Hg}$ & $99.44(15)$ \\
\hline
\end{tabular}




\begin{tabular}{|c|c|}
\hline $\mathrm{C}(17)-\mathrm{O}(1)-\mathrm{C}(18)$ & $105.0(4)$ \\
\hline $\mathrm{C}(27)-\mathrm{O}(2)-\mathrm{C}(28)$ & $104.4(5)$ \\
\hline $\mathrm{C}(17)-\mathrm{N}(1)-\mathrm{C}(19)$ & $108.2(4)$ \\
\hline $\mathrm{C}(17)-\mathrm{N}(1)-\mathrm{Hg}$ & $132.1(4)$ \\
\hline $\mathrm{C}(19)-\mathrm{N}(1)-\mathrm{Hg}$ & $119.7(3)$ \\
\hline $\mathrm{C}(27)-\mathrm{N}(2)-\mathrm{C}(29)$ & $107.2(5)$ \\
\hline$C(12)-C(11)-C(16)$ & $119.1(5)$ \\
\hline $\mathrm{C}(12)-\mathrm{C}(11)-\mathrm{Se}$ & $112.5(4)$ \\
\hline $\mathrm{C}(16)-\mathrm{C}(11)-\mathrm{Se}$ & $128.4(4)$ \\
\hline $\mathrm{C}(11)-\mathrm{C}(12)-\mathrm{C}(13)$ & $121.6(6)$ \\
\hline$C(14)-C(13)-C(12)$ & $119.7(6)$ \\
\hline$C(13)-C(14)-C(15)$ & $119.9(6)$ \\
\hline$C(14)-C(15)-C(16)$ & $121.5(6)$ \\
\hline$C(15)-C(16)-C(11)$ & $118.3(5)$ \\
\hline$C(15)-C(16)-C(17)$ & $117.8(5)$ \\
\hline$C(11)-C(16)-C(17)$ & $123.9(5)$ \\
\hline $\mathrm{N}(1)-\mathrm{C}(17)-\mathrm{O}(1)$ & $117.2(5)$ \\
\hline $\mathrm{N}(1)-\mathrm{C}(17)-\mathrm{C}(16)$ & $130.2(5)$ \\
\hline $\mathrm{O}(1)-\mathrm{C}(17)-\mathrm{C}(16)$ & $112.5(5)$ \\
\hline $\mathrm{O}(1)-\mathrm{C}(18)-\mathrm{C}(19)$ & $105.2(5)$ \\
\hline $\mathrm{C}(110)-\mathrm{C}(19)-\mathrm{N}(1)$ & $109.0(5)$ \\
\hline $\mathrm{C}(110)-\mathrm{C}(19)-\mathrm{C}(111)$ & $111.3(6)$ \\
\hline $\mathrm{N}(1)-\mathrm{C}(19)-\mathrm{C}(111)$ & $110.0(5)$ \\
\hline
\end{tabular}




\begin{tabular}{|c|c|}
\hline $\mathrm{C}(110)-\mathrm{C}(19)-\mathrm{C}(18)$ & $112.6(6)$ \\
\hline $\mathrm{N}(1)-\mathrm{C}(19)-\mathrm{C}(18)$ & $101.3(5)$ \\
\hline $\mathrm{C}(111)-\mathrm{C}(19)-\mathrm{C}(18)$ & $112.0(5)$ \\
\hline$C(22)-C(21)-C(26)$ & $119.1(5)$ \\
\hline $\mathrm{C}(22)-\mathrm{C}(21)-\mathrm{Se}$ & $121.4(4)$ \\
\hline$C(26)-C(21)-S e$ & $119.5(4)$ \\
\hline$C(21)-C(22)-C(23)$ & $120.5(5)$ \\
\hline$C(24)-C(23)-C(22)$ & $120.4(5)$ \\
\hline $\mathrm{C}(23)-\mathrm{C}(24)-\mathrm{C}(25)$ & $119.9(5)$ \\
\hline$C(24)-C(25)-C(26)$ & $121.0(5)$ \\
\hline$C(25)-C(26)-C(21)$ & $119.0(5)$ \\
\hline$C(25)-C(26)-C(27)$ & $120.3(5)$ \\
\hline$C(21)-C(26)-C(27)$ & $120.6(5)$ \\
\hline $\mathrm{N}(2)-\mathrm{C}(27)-\mathrm{O}(2)$ & $118.7(5)$ \\
\hline $\mathrm{N}(2)-\mathrm{C}(27)-\mathrm{C}(26)$ & $125.3(5)$ \\
\hline $\mathrm{O}(2)-\mathrm{C}(27)-\mathrm{C}(26)$ & $115.9(5)$ \\
\hline $\mathrm{O}(2)-\mathrm{C}(28)-\mathrm{C}(29)$ & $106.0(5)$ \\
\hline $\mathrm{N}(2)-\mathrm{C}(29)-\mathrm{C}(210)$ & $107.8(6)$ \\
\hline $\mathrm{N}(2)-\mathrm{C}(29)-\mathrm{C}(211)$ & $109.5(6)$ \\
\hline$C(210)-C(29)-C(211)$ & $110.7(8)$ \\
\hline $\mathrm{N}(2)-\mathrm{C}(29)-\mathrm{C}(28)$ & $102.6(5)$ \\
\hline $\mathrm{C}(210)-\mathrm{C}(29)-\mathrm{C}(28)$ & $113.5(8)$ \\
\hline $\mathrm{C}(211)-\mathrm{C}(29)-\mathrm{C}(28)$ & $112.3(7)$ \\
\hline
\end{tabular}


Table 4. Anisotropic displacement parameters $\left(\AA^{2} \times 10^{3}\right)$ for 5 . The anisotropic displacement factor exponent takes the form: $-2 \pi^{2}\left[h^{2} a^{* 2} U^{11}+\ldots+2 h k a^{*} b^{*} U^{12}\right]$

\begin{tabular}{|c|c|c|c|c|c|c|}
\hline & $\mathrm{U}^{11}$ & $\mathrm{U}^{22}$ & $\mathrm{U}^{33}$ & $\mathrm{U}^{23}$ & $\mathrm{U}^{13}$ & $\mathrm{U}^{12}$ \\
\hline $\mathrm{Hg}$ & $58(1)$ & $44(1)$ & $41(1)$ & $-5(1)$ & $19(1)$ & $-1(1)$ \\
\hline $\mathrm{Se}$ & $35(1)$ & $33(1)$ & $39(1)$ & $4(1)$ & $11(1)$ & $-1(1)$ \\
\hline $\mathrm{Cl}(1)$ & $90(1)$ & $71(1)$ & $60(1)$ & $22(1)$ & $36(1)$ & $27(1)$ \\
\hline $\mathrm{Cl}(2)$ & $93(1)$ & $40(1)$ & $97(2)$ & $-15(1)$ & $30(1)$ & $-1(1)$ \\
\hline $\mathrm{O}(1)$ & $49(2)$ & $56(3)$ & $54(3)$ & $-12(2)$ & $27(2)$ & $-6(2)$ \\
\hline $\mathrm{O}(2)$ & $70(3)$ & $36(2)$ & $61(3)$ & $10(2)$ & $33(2)$ & $16(2)$ \\
\hline $\mathrm{N}(1)$ & $36(2)$ & $29(2)$ & $37(2)$ & $-4(2)$ & $11(2)$ & $-6(2)$ \\
\hline $\mathrm{N}(2)$ & $50(3)$ & $36(3)$ & $44(3)$ & $5(2)$ & $19(2)$ & $8(2)$ \\
\hline $\mathrm{C}(11)$ & $43(3)$ & $27(3)$ & $34(3)$ & $2(2)$ & $3(2)$ & $-2(2)$ \\
\hline$C(12)$ & $55(4)$ & $37(3)$ & $55(4)$ & $0(3)$ & $9(3)$ & $-8(3)$ \\
\hline$C(13)$ & $84(5)$ & $27(3)$ & $68(5)$ & $-3(3)$ & $10(4)$ & $-10(4)$ \\
\hline $\mathrm{C}(14)$ & $85(5)$ & $29(3)$ & $69(5)$ & $-1(3)$ & $13(4)$ & $15(4)$ \\
\hline$C(15)$ & $59(4)$ & $40(3)$ & $54(4)$ & $-2(3)$ & $16(3)$ & $14(3)$ \\
\hline$C(16)$ & $50(3)$ & $31(3)$ & $29(3)$ & $2(2)$ & $8(2)$ & $3(2)$ \\
\hline$C(17)$ & $39(3)$ & $35(3)$ & $36(3)$ & $4(2)$ & $13(2)$ & $3(2)$ \\
\hline $\mathrm{C}(18)$ & $52(4)$ & $59(4)$ & $64(4)$ & $-12(4)$ & $27(4)$ & $-15(3)$ \\
\hline$C(19)$ & $35(3)$ & $44(3)$ & $40(3)$ & $2(2)$ & $11(2)$ & $-7(2)$ \\
\hline $\mathrm{C}(110)$ & $69(5)$ & $49(4)$ & $70(5)$ & $10(4)$ & $16(4)$ & $-22(4)$ \\
\hline
\end{tabular}




$\begin{array}{lllllll}\mathrm{C}(111) & 57(4) & 67(5) & 50(4) & -8(4) & 9(3) & -9(4) \\ \mathrm{C}(21) & 31(3) & 35(3) & 31(3) & 1(2) & 6(2) & 0(2) \\ \mathrm{C}(22) & 48(3) & 44(3) & 35(3) & 2(3) & 10(3) & 12(3) \\ \mathrm{C}(23) & 52(3) & 61(4) & 33(3) & 7(3) & 17(3) & 13(3) \\ \mathrm{C}(24) & 51(3) & 55(4) & 33(3) & 13(3) & 13(3) & 2(3) \\ \mathrm{C}(25) & 48(3) & 34(3) & 40(3) & 7(2) & 10(3) & 6(3) \\ \mathrm{C}(26) & 33(3) & 35(3) & 31(3) & -2(2) & 3(2) & 0(2) \\ \mathrm{C}(27) & 33(3) & 36(3) & 42(3) & 0(2) & 8(2) & 5(2) \\ \mathrm{C}(28) & 100(6) & 50(4) & 77(5) & -1(4) & 57(5) & 17(4) \\ \mathrm{C}(29) & 56(4) & 47(4) & 54(4) & -1(3) & 33(3) & 6(3) \\ \mathrm{C}(210) & 60(5) & 87(8) & 178(13) & -43(7) & 61(6) & -6(5) \\ \mathrm{C}(211) & 134(9) & 102(8) & 58(5) & 3(5) & 50(6) & 32(6)\end{array}$

Table 5. Hydrogen coordinates ( $\left.\mathrm{x} 10^{4}\right)$ and isotropic displacement parameters $\left(\AA^{2} \mathrm{x} 10^{3}\right)$ for 5 .

\begin{tabular}{lrrrr}
\hline & \multicolumn{1}{c}{ x } & $\mathrm{z}$ & $\mathrm{U}(\mathrm{eq})$ \\
$\mathrm{H}(12 \mathrm{~A})$ & 6072 & -2657 & 951 & $70(20)$ \\
$\mathrm{H}(13 \mathrm{~A})$ & 6962 & -4514 & 812 & $70(20)$ \\
$\mathrm{H}(14 \mathrm{~A})$ & 8783 & -4458 & 785 & $48(17)$ \\
$\mathrm{H}(15 \mathrm{~A})$ & 9713 & -2556 & 908 & $60(20)$ \\
$\mathrm{H}(18 \mathrm{~A})$ & 10874 & 1198 & 697 & $80(20)$
\end{tabular}




\begin{tabular}{|c|c|c|c|c|}
\hline $\mathrm{H}(18 \mathrm{~B})$ & 11337 & 514 & 1465 & $110(30)$ \\
\hline $\mathrm{H}(11 \mathrm{~A})$ & 9180 & 2362 & 428 & $60(20)$ \\
\hline $\mathrm{H}(11 \mathrm{~B})$ & 9975 & 3336 & 941 & $57(19)$ \\
\hline $\mathrm{H}(11 \mathrm{C})$ & 8831 & 3013 & 1076 & $100(30)$ \\
\hline $\mathrm{H}(11 \mathrm{D})$ & 10852 & 1412 & 2505 & $60(20)$ \\
\hline $\mathrm{H}(11 \mathrm{E})$ & 10129 & 2646 & 2369 & $60(20)$ \\
\hline $\mathrm{H}(11 \mathrm{~F})$ & 11227 & 2594 & 2131 & $170(50)$ \\
\hline $\mathrm{H}(22 \mathrm{~A})$ & 7264 & -178 & -151 & $47(17)$ \\
\hline $\mathrm{H}(23 \mathrm{~A})$ & 7406 & 1210 & -1073 & $43(16)$ \\
\hline $\mathrm{H}(24 \mathrm{~A})$ & 6840 & 3279 & -1062 & $38(15)$ \\
\hline $\mathrm{H}(25 \mathrm{~A})$ & 5918 & 3913 & -224 & $42(16)$ \\
\hline $\mathrm{H}(28 \mathrm{~A})$ & 3787 & 4378 & 1162 & $70(20)$ \\
\hline $\mathrm{H}(28 \mathrm{~B})$ & 4941 & 4589 & 1745 & $150(40)$ \\
\hline $\mathrm{H}(21 \mathrm{~A})$ & 3070 & 2148 & 904 & $170(60)$ \\
\hline $\mathrm{H}(21 \mathrm{~B})$ & 2836 & 2652 & 1629 & $120(30)$ \\
\hline $\mathrm{H}(21 \mathrm{C})$ & 3352 & 1301 & 1615 & $150(50)$ \\
\hline H(21D) & 5694 & 2824 & 2624 & $120(40)$ \\
\hline $\mathrm{H}(21 \mathrm{E})$ & 4775 & 1846 & 2668 & $80(30)$ \\
\hline$H(21 F)$ & 4558 & 3305 & 2712 & $110(30)$ \\
\hline
\end{tabular}


Table 1. Crystal data and structure refinement for 7.

Empirical formula

Formula weight

Temperature

Wavelength

Crystal system, space group

Unit cell dimensions

Volume

Z, Calculated density

Absorption coefficient

$\mathrm{F}(000)$

Crystal size

Theta range for data collection

Limiting indices

Reflections collected / unique

Completeness to theta $=27.50$

Absorption correction

Max. and min. transmission

Refinement method

Data / restraints / parameters
C11 H12 Cl N O Te

293(2) K

$0.71073 \AA$

Monoclinic, P2(1)/n

$$
\begin{aligned}
& \mathrm{a}=11.5638(15) \AA \quad \text { alpha }=90 \mathrm{deg} . \\
& \mathrm{b}=9.1432(11) \AA \quad \text { beta }=113.481(9) \mathrm{deg} . \\
& \mathrm{c}=12.7691(11) \AA \quad \text { gamma }=90 \mathrm{deg} .
\end{aligned}
$$

$1238.3(2) \AA^{3}$

4, $1.809 \mathrm{Mg} / \mathrm{m}^{3}$

$2.592 \mathrm{~mm}^{-1}$

648

$0.22 \times 0.66 \times 0.27 \mathrm{~mm}$

2.01 to $27.50 \mathrm{deg}$.

$0<=\mathrm{h}<=15,-11<=\mathrm{k}<=0,-16<=\mathrm{l}<=15$

$2976 / 2840[\mathrm{R}($ int $)=0.0169]$

$100.0 \%$

\section{SHELXA}

0.8545 and 0.5331

Full-matrix least-squares on $\mathrm{F}^{2}$

2840 / 0 / 151 
Goodness-of-fit on $\mathrm{F}^{2}$

Final $\mathrm{R}$ indices [I $>2$ sigma(I)]

$\mathrm{R}$ indices (all data)

Extinction coefficient

Largest diff. peak and hole
1.105

$\mathrm{R} 1=0.0272, \mathrm{wR} 2=0.0680$

$\mathrm{R} 1=0.0334, \mathrm{wR} 2=0.0707$

$0.0096(6)$

0.540 and -0.415 e. $\AA^{-3}$

Table 2. Atomic coordinates $\left(\mathrm{x} 10^{4}\right)$ and equivalent isotropic displacement parameters $\left(\AA^{2} \mathrm{x}\right.$ $10^{3}$ ) for $7 . \quad U(e q)$ is defined as one third of the trace of the orthogonalized $U^{i j}$ tensor.

\begin{tabular}{|c|c|c|c|c|}
\hline & $\mathrm{x}$ & $\mathrm{y}$ & $\mathrm{z}$ & $\mathrm{J}(\mathrm{eq})$ \\
\hline $\mathrm{Te}$ & $8451(1)$ & $9480(1)$ & $283(1)$ & $43(1)$ \\
\hline $\mathrm{Cl}$ & $9697(1)$ & $11753(1)$ & $1282(1)$ & $68(1)$ \\
\hline $\mathrm{O}(1)$ & $5433(3)$ & $6484(3)$ & $-283(2)$ & $61(1)$ \\
\hline $\mathrm{N}(1)$ & $7131(3)$ & $7579(3)$ & $-342(2)$ & $46(1)$ \\
\hline $\mathrm{C}(1)$ & $7180(3)$ & $9766(3)$ & $1079(2)$ & $39(1)$ \\
\hline$C(2)$ & $7204(3)$ & $10899(4)$ & 1813(3) & $49(1)$ \\
\hline$C(3)$ & $6315(4)$ & $10947(4)$ & $2277(3)$ & $56(1)$ \\
\hline $\mathrm{C}(4)$ & $5388(4)$ & $9893(4)$ & $2030(3)$ & $54(1)$ \\
\hline$C(5)$ & $5335(3)$ & $8772(4)$ & $1294(3)$ & $49(1)$ \\
\hline$C(6)$ & $6239(3)$ & $8704(3)$ & $821(2)$ & $41(1)$ \\
\hline$C(7)$ & $6272(3)$ & $7575(3)$ & $49(3)$ & $45(1)$ \\
\hline$C(8)$ & $5737(6)$ & $5636(5)$ & $-1115(5)$ & $88(2)$ \\
\hline
\end{tabular}




$\begin{array}{lllll}\mathrm{C}(9) & 6992(4) & 6273(4) & -1078(3) & 55(1) \\ \mathrm{C}(10) & 6878(4) & 6743(5) & -2248(3) & 66(1) \\ \mathrm{C}(11) & 8139(7) & 5287(6) & -518(5) & 100(2)\end{array}$

Table 3. Anisotropic displacement parameters $\left(\AA^{2} \times 10^{3}\right)$ for 7 .

The anisotropic displacement factor exponent takes the form: $-2 \pi^{2}\left[h^{2} a^{* 2} U^{11}+\ldots+2 h k a^{*}\right.$ $\left.\mathrm{b}^{*} \mathrm{U}^{12}\right]$

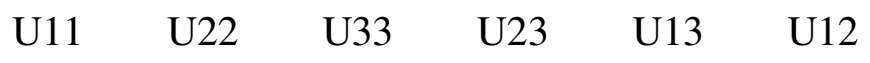

$\begin{array}{lllllll}\mathrm{Te} & 47(1) & 46(1) & 41(1) & -4(1) & 21(1) & -1(1) \\ \mathrm{Cl} & 70(1) & 60(1) & 83(1) & -23(1) & 42(1) & -19(1) \\ \mathrm{O}(1) & 85(2) & 47(1) & 69(2) & -15(1) & 48(2) & -21(1) \\ \mathrm{N}(1) & 57(2) & 40(1) & 45(1) & -8(1) & 24(1) & -4(1) \\ \mathrm{C}(1) & 45(2) & 38(2) & 36(1) & 1(1) & 17(1) & 3(1) \\ \mathrm{C}(2) & 55(2) & 47(2) & 46(2) & -10(1) & 21(2) & -4(2) \\ \mathrm{C}(3) & 65(2) & 55(2) & 53(2) & -11(2) & 28(2) & 6(2) \\ \mathrm{C}(4) & 66(2) & 54(2) & 56(2) & 4(2) & 39(2) & 9(2) \\ \mathrm{C}(5) & 58(2) & 44(2) & 52(2) & 8(2) & 29(2) & 3(2) \\ \mathrm{C}(6) & 51(2) & 37(2) & 38(1) & 5(1) & 21(1) & 3(1) \\ \mathrm{C}(7) & 57(2) & 37(2) & 41(2) & 2(1) & 22(1) & -2(1) \\ \mathrm{C}(8) & 135(5) & 68(3) & 98(4) & -38(3) & 85(4) & -44(3)\end{array}$




$$
\begin{array}{lllllll}
\mathrm{C}(9) & 74(2) & 43(2) & 54(2) & -15(2) & 34(2) & -8(2) \\
\mathrm{C}(10) & 70(3) & 81(3) & 51(2) & -20(2) & 29(2) & -21(2) \\
\mathrm{C}(11) & 138(6) & 64(3) & 89(4) & -11(3) & 36(4) & 31(4)
\end{array}
$$

\begin{tabular}{|c|c|c|c|c|}
\hline & $\mathrm{x}$ & $\mathrm{y}$ & z & $\mathrm{U}(\mathrm{eq})$ \\
\hline $\mathrm{H}(2 \mathrm{~A})$ & 7819 & 11621 & 1991 & $68(12)$ \\
\hline $\mathrm{H}(3 \mathrm{~A})$ & 6339 & 11709 & 2768 & $73(12)$ \\
\hline $\mathrm{H}(4 \mathrm{~A})$ & 4803 & 9941 & 2360 & $59(11)$ \\
\hline $\mathrm{H}(5 \mathrm{~A})$ & 4706 & 8066 & 1112 & $48(9)$ \\
\hline $\mathrm{H}(8 \mathrm{~A})$ & 5076 & 5737 & -1874 & $160(30)$ \\
\hline $\mathrm{H}(8 \mathrm{~B})$ & 5833 & 4608 & -910 & $130(20)$ \\
\hline $\mathrm{H}(10 \mathrm{~A})$ & 7650 & 7198 & -2189 & $89(15)$ \\
\hline $\mathrm{H}(10 \mathrm{~B})$ & 6197 & 7427 & -2563 & $88(16)$ \\
\hline $\mathrm{H}(10 \mathrm{C})$ & 6715 & 5903 & -2737 & $106(18)$ \\
\hline $\mathrm{H}(11 \mathrm{~A})$ & 8889 & 5813 & -435 & $120(30)$ \\
\hline $\mathrm{H}(11 \mathrm{~B})$ & 8057 & 4440 & -986 & $85(15)$ \\
\hline $\mathrm{H}(11 \mathrm{C})$ & 8193 & 4987 & 221 & $120(20)$ \\
\hline
\end{tabular}

Table 4. Hydrogen coordinates ( x 104) and isotropic displacement parameters $\left(\AA^{2} \times 10^{3}\right)$ for 7 . 
Table 5. Bond lengths $[\AA]$ and angles $\left[{ }^{\circ}\right]$ for 7 .

\begin{tabular}{|c|c|}
\hline $\mathrm{Te}-\mathrm{C}(1)$ & $2.110(3)$ \\
\hline $\mathrm{Te}-\mathrm{N}(1)$ & $2.241(3)$ \\
\hline $\mathrm{Te}-\mathrm{Cl}$ & $2.5602(10)$ \\
\hline $\mathrm{O}(1)-\mathrm{C}(7)$ & $1.338(4)$ \\
\hline $\mathrm{O}(1)-\mathrm{C}(8)$ & $1.467(5)$ \\
\hline $\mathrm{N}(1)-\mathrm{C}(7)$ & $1.276(4)$ \\
\hline $\mathrm{N}(1)-\mathrm{C}(9)$ & $1.488(4)$ \\
\hline $\mathrm{C}(1)-\mathrm{C}(2)$ & $1.390(4)$ \\
\hline$C(1)-C(6)$ & $1.396(4)$ \\
\hline$C(2)-C(3)$ & $1.376(5)$ \\
\hline$C(3)-C(4)$ & $1.381(6)$ \\
\hline$C(4)-C(5)$ & $1.376(5)$ \\
\hline$C(5)-C(6)$ & $1.401(4)$ \\
\hline$C(6)-C(7)$ & $1.439(4)$ \\
\hline $\mathrm{C}(8)-\mathrm{C}(9)$ & $1.547(6)$ \\
\hline $\mathrm{C}(9)-\mathrm{C}(10)$ & $1.509(5)$ \\
\hline $\mathrm{C}(9)-\mathrm{C}(11)$ & $1.526(7)$ \\
\hline $\mathrm{C}(1)-\mathrm{Te}-\mathrm{N}(1)$ & $76.17(10)$ \\
\hline $\mathrm{C}(1)-\mathrm{Te}-\mathrm{Cl}$ & $91.22(9)$ \\
\hline $\mathrm{N}(1)-\mathrm{Te}-\mathrm{Cl}$ & $167.29(7)$ \\
\hline $\mathrm{C}(7)-\mathrm{O}(1)-\mathrm{C}(8)$ & $105.7(3)$ \\
\hline
\end{tabular}




\begin{tabular}{|c|c|}
\hline $\mathrm{C}(7)-\mathrm{N}(1)-\mathrm{C}(9)$ & $109.9(3)$ \\
\hline $\mathrm{C}(7)-\mathrm{N}(1)-\mathrm{Te}$ & $112.9(2)$ \\
\hline $\mathrm{C}(9)-\mathrm{N}(1)-\mathrm{Te}$ & $137.2(2)$ \\
\hline $\mathrm{C}(2)-\mathrm{C}(1)-\mathrm{C}(6)$ & $118.8(3)$ \\
\hline $\mathrm{C}(2)-\mathrm{C}(1)-\mathrm{Te}$ & $125.9(2)$ \\
\hline $\mathrm{C}(6)-\mathrm{C}(1)-\mathrm{Te}$ & $115.3(2)$ \\
\hline $\mathrm{C}(3)-\mathrm{C}(2)-\mathrm{C}(1)$ & $119.8(3)$ \\
\hline $\mathrm{C}(2)-\mathrm{C}(3)-\mathrm{C}(4)$ & $121.6(3)$ \\
\hline $\mathrm{C}(5)-\mathrm{C}(4)-\mathrm{C}(3)$ & $119.7(3)$ \\
\hline $\mathrm{C}(4)-\mathrm{C}(5)-\mathrm{C}(6)$ & $119.3(3)$ \\
\hline $\mathrm{C}(1)-\mathrm{C}(6)-\mathrm{C}(5)$ & $120.8(3)$ \\
\hline $\mathrm{C}(1)-\mathrm{C}(6)-\mathrm{C}(7)$ & $115.5(3)$ \\
\hline$C(5)-C(6)-C(7)$ & $123.7(3)$ \\
\hline $\mathrm{N}(1)-\mathrm{C}(7)-\mathrm{O}(1)$ & $117.2(3)$ \\
\hline $\mathrm{N}(1)-\mathrm{C}(7)-\mathrm{C}(6)$ & $120.2(3)$ \\
\hline $\mathrm{O}(1)-\mathrm{C}(7)-\mathrm{C}(6)$ & $122.6(3)$ \\
\hline $\mathrm{O}(1)-\mathrm{C}(8)-\mathrm{C}(9)$ & $105.8(3)$ \\
\hline $\mathrm{N}(1)-\mathrm{C}(9)-\mathrm{C}(10)$ & $110.0(3)$ \\
\hline $\mathrm{N}(1)-\mathrm{C}(9)-\mathrm{C}(11)$ & $108.9(3)$ \\
\hline$C(10)-C(9)-C(11)$ & $110.4(4)$ \\
\hline $\mathrm{N}(1)-\mathrm{C}(9)-\mathrm{C}(8)$ & $100.7(3)$ \\
\hline $\mathrm{C}(10)-\mathrm{C}(9)-\mathrm{C}(8)$ & $111.5(4)$ \\
\hline $\mathrm{C}(11)-\mathrm{C}(9)-\mathrm{C}(8)$ & $114.9(4)$ \\
\hline
\end{tabular}


Table 1. Crystal data and structure refinement for $\mathbf{8}$.

Empirical formula

Formula weight

Temperature

Wavelength

Crystal system, space group

Unit cell dimensions

Volume

Z, Calculated density

Absorption coefficient

$\mathrm{F}(000)$

Crystal size

Theta range for data collection

Limiting indices

Reflections collected / unique

Completeness to theta $=27.50$

Absorption correction

Max. and min. transmission

Refinement method

Data / restraints / parameters

Goodness-of-fit on $\mathrm{F}^{2}$
$\mathrm{C} 11 \mathrm{H} 12 \mathrm{Cl} \mathrm{Hg} \mathrm{N} \mathrm{O}$

410.26

293(2) K

$0.71073 \AA$

Monoclinic, P2(1)/m

$$
\begin{aligned}
& \mathrm{a}=20.2339(16) \AA \quad \text { alpha }=90 \mathrm{deg} . \\
& \mathrm{b}=6.9091(8) \AA \quad \text { beta }=100.962(8) \mathrm{deg} . \\
& \mathrm{c}=8.6311(9) \AA \quad \text { gamma }=90 \mathrm{deg} .
\end{aligned}
$$

1184.6(2) $\AA^{3}$

4, $2.300 \mathrm{Mg} / \mathrm{m}^{3}$

$13.191 \mathrm{~mm}^{-1}$

760

$0.10 \times 0.88 \times 0.26 \mathrm{~mm}$

2.40 to $27.50 \mathrm{deg}$.

$0<=\mathrm{h}<=26,-8<=\mathrm{k}<=0,-11<=\mathrm{l}<=11$

$3004 / 2930[\mathrm{R}($ int $)=0.0359]$

$99.4 \%$

SHELXA

0.1561 and 0.6286

Full-matrix least-squares on $\mathrm{F}^{2}$

$2930 / 0 / 192$

1.159 
Final $\mathrm{R}$ indices $[\mathrm{I}>2 \operatorname{sigma}(\mathrm{I})] \quad \mathrm{R} 1=0.0559, \mathrm{wR} 2=0.1406$

$\mathrm{R}$ indices (all data) $\quad \mathrm{R} 1=0.0745, \mathrm{wR} 2=0.1494$

Extinction coefficient $\quad 0.0023(3)$

Largest diff. peak and hole $\quad 2.140$ and -2.334 e. $\AA^{-3}$

Table 2. Atomic coordinates $\left(\mathrm{x} 10^{4}\right)$ and equivalent isotropic displacement parameters $\left(\AA^{2} \times 10^{3}\right)$ for 8 . U(eq) is defined as one third of the trace of the orthogonalized $U^{i j}$ tensor.

\begin{tabular}{|c|c|c|c|c|}
\hline & $\mathrm{x}$ & $\mathrm{y}$ & \multicolumn{2}{|c|}{$\mathrm{U}(\mathrm{eq})$} \\
\hline $\operatorname{Hg}(1)$ & $4062(1)$ & -2500 & $-798(1)$ & $41(1)$ \\
\hline $\mathrm{Hg}(2)$ & 11002(1) & -7500 & $5053(1)$ & $40(1)$ \\
\hline $\mathrm{Cl}(1)$ & 2953(3) & -2500 & $-2031(8)$ & $70(2)$ \\
\hline $\mathrm{Cl}(2)$ & $12109(2)$ & -7500 & $4776(8)$ & $63(2)$ \\
\hline $\mathrm{O}(11)$ & 4994(6) & -2500 & $4362(15)$ & $50(4)$ \\
\hline $\mathrm{O}(21)$ & $10078(6)$ & -7500 & $9355(16)$ & $51(4)$ \\
\hline $\mathrm{N}(11)$ & $4122(8)$ & -2500 & $2290(20)$ & $44(4)$ \\
\hline $\mathrm{N}(21)$ & 10927(6) & -7500 & $8039(16)$ & $33(3)$ \\
\hline $\mathrm{C}(11)$ & $5086(8)$ & -2500 & 171(19) & $28(3)$ \\
\hline$C(12)$ & $5573(8)$ & -2500 & $-840(20)$ & $39(4)$ \\
\hline$C(13)$ & $6244(10)$ & -2500 & $-90(30)$ & $51(5)$ \\
\hline$C(14)$ & $6428(9)$ & -2500 & $1490(30)$ & $48(5)$ \\
\hline$C(15)$ & 5960(9) & -2500 & $2540(20)$ & $48(5)$ \\
\hline
\end{tabular}




$\begin{array}{lrlrr}\mathrm{C}(16) & 5291(9) & -2500 & 1810(20) & 38(4) \\ \mathrm{C}(17) & 4751(9) & -2500 & 2790(20) & 43(5) \\ \mathrm{C}(18) & 4335(13) & -2500 & 5080(20) & 56(6) \\ \mathrm{C}(19) & 3820(9) & -2500 & 3640(20) & 38(4) \\ \mathrm{C}(110) & 3380(10) & -4350(30) & 3630(20) & 72(5) \\ \mathrm{C}(21) & 9983(10) & -7500 & 5080(30) & 42(4) \\ \mathrm{C}(22) & 9481(8) & -7500 & 3740(30) & 47(5) \\ \mathrm{C}(23) & 8818(11) & -7500 & 3800(20) & 53(6) \\ \mathrm{C}(24) & 8631(9) & -7500 & 5270(30) & 50(5) \\ \mathrm{C}(25) & 9089(9) & -7500 & 6570(30) & 43(5) \\ \mathrm{C}(26) & 9782(7) & -7500 & 6580(20) & 31(4) \\ \mathrm{C}(27) & 10291(9) & -7500 & 7990(20) & 37(4) \\ \mathrm{C}(28) & 10635(11) & -7500 & 10620(20) & 55(6) \\ \mathrm{C}(29) & 11246(9) & -7500 & 9760(20) & 37(4) \\ \mathrm{C}(210) & 11683(9) & -5720(30) & 10100(20) & 74(6)\end{array}$

Table 3. Bond lengths $[\AA]$ and angles [deg] for $\mathbf{8}$.

\begin{tabular}{ll}
\hline $\mathrm{Hg}(1)-\mathrm{C}(11)$ & $2.081(16)$ \\
$\mathrm{Hg}(1)-\mathrm{Cl}(1)$ & $2.293(5)$ \\
$\mathrm{Hg}(1)-\mathrm{N}(11)$ & $2.645(17)$ \\
$\mathrm{Hg}(2)-\mathrm{C}(21)$ & $2.065(19)$
\end{tabular}




\begin{tabular}{|c|c|}
\hline $\mathrm{Hg}(2)-\mathrm{Cl}(2)$ & $2.299(5)$ \\
\hline $\mathrm{Hg}(2)-\mathrm{N}(21)$ & $2.610(14)$ \\
\hline $\mathrm{O}(11)-\mathrm{C}(17)$ & $1.35(2)$ \\
\hline $\mathrm{O}(11)-\mathrm{C}(18)$ & $1.57(3)$ \\
\hline $\mathrm{O}(21)-\mathrm{C}(27)$ & $1.332(19)$ \\
\hline $\mathrm{O}(21)-\mathrm{C}(28)$ & $1.41(3)$ \\
\hline $\mathrm{N}(11)-\mathrm{C}(17)$ & $1.26(2)$ \\
\hline N(11)-C(19) & $1.41(2)$ \\
\hline $\mathrm{N}(21)-\mathrm{C}(27)$ & $1.28(2)$ \\
\hline $\mathrm{N}(21)-\mathrm{C}(29)$ & $1.50(2)$ \\
\hline $\mathrm{C}(11)-\mathrm{C}(16)$ & $1.39(2)$ \\
\hline $\mathrm{C}(11)-\mathrm{C}(12)$ & $1.44(2)$ \\
\hline$C(12)-C(13)$ & $1.39(3)$ \\
\hline$C(13)-C(14)$ & $1.35(3)$ \\
\hline$C(14)-C(15)$ & $1.43(3)$ \\
\hline$C(15)-C(16)$ & $1.38(2)$ \\
\hline$C(16)-C(17)$ & $1.51(3)$ \\
\hline $\mathrm{C}(18)-\mathrm{C}(19)$ & $1.46(3)$ \\
\hline C(19)-C(110)\#1 & $1.55(2)$ \\
\hline$C(19)-C(110)$ & $1.55(2)$ \\
\hline $\mathrm{C}(21)-\mathrm{C}(22)$ & $1.38(3)$ \\
\hline $\mathrm{C}(21)-\mathrm{C}(26)$ & $1.43(2)$ \\
\hline $\mathrm{C}(22)-\mathrm{C}(23)$ & $1.35(3)$ \\
\hline
\end{tabular}




\begin{tabular}{|c|c|}
\hline $\mathrm{C}(23)-\mathrm{C}(24)$ & $1.39(3)$ \\
\hline $\mathrm{C}(24)-\mathrm{C}(25)$ & $1.31(3)$ \\
\hline$C(25)-C(26)$ & $1.40(2)$ \\
\hline$C(26)-C(27)$ & $1.43(3)$ \\
\hline $\mathrm{C}(28)-\mathrm{C}(29)$ & $1.56(3)$ \\
\hline$C(29)-C(210)$ & $1.51(2)$ \\
\hline $\mathrm{C}(29)-\mathrm{C}(210) \# 2$ & $1.51(2)$ \\
\hline $\mathrm{C}(11)-\mathrm{Hg}(1)-\mathrm{Cl}(1)$ & $176.1(5)$ \\
\hline $\mathrm{C}(11)-\mathrm{Hg}(1)-\mathrm{N}(11)$ & $75.2(5)$ \\
\hline $\mathrm{Cl}(1)-\mathrm{Hg}(1)-\mathrm{N}(11)$ & $108.7(4)$ \\
\hline $\mathrm{C}(21)-\mathrm{Hg}(2)-\mathrm{Cl}(2)$ & $174.7(6)$ \\
\hline $\mathrm{C}(21)-\mathrm{Hg}(2)-\mathrm{N}(21)$ & $75.2(6)$ \\
\hline $\mathrm{Cl}(2)-\mathrm{Hg}(2)-\mathrm{N}(21)$ & $110.1(3)$ \\
\hline $\mathrm{C}(17)-\mathrm{O}(11)-\mathrm{C}(18)$ & $102.8(13)$ \\
\hline $\mathrm{C}(27)-\mathrm{O}(21)-\mathrm{C}(28)$ & $109.7(15)$ \\
\hline $\mathrm{C}(17)-\mathrm{N}(11)-\mathrm{C}(19)$ & $106.5(17)$ \\
\hline $\mathrm{C}(17)-\mathrm{N}(11)-\mathrm{Hg}(1)$ & $101.2(13)$ \\
\hline $\mathrm{C}(19)-\mathrm{N}(11)-\mathrm{Hg}(1)$ & $152.3(12)$ \\
\hline $\mathrm{C}(27)-\mathrm{N}(21)-\mathrm{C}(29)$ & $105.9(14)$ \\
\hline $\mathrm{C}(27)-\mathrm{N}(21)-\mathrm{Hg}(2)$ & $102.2(11)$ \\
\hline $\mathrm{C}(29)-\mathrm{N}(21)-\mathrm{Hg}(2)$ & $151.9(10)$ \\
\hline$C(16)-C(11)-C(12)$ & $120.7(16)$ \\
\hline
\end{tabular}




$$
\begin{aligned}
& \mathrm{C}(16)-\mathrm{C}(11)-\mathrm{Hg}(1) \quad 119.3(12) \\
& \text { C(12)-C(11)-Hg(1) 120.1(12) } \\
& \text { C(13)-C(12)-C(11) 116.1(17) } \\
& \text { C(14)-C(13)-C(12) 122.0(18) } \\
& \text { C(13)-C(14)-C(15) 123.8(18) } \\
& \text { C(16)-C(15)-C(14) 114.5(18) } \\
& \text { C(15)-C(16)-C(11) 123.0(18) } \\
& \text { C(15)-C(16)-C(17) 119.5(17) } \\
& \text { C(11)-C(16)-C(17) 117.6(16) } \\
& \text { N(11)-C(17)-O(11) } \quad 119.5(17) \\
& \text { N(11)-C(17)-C(16) 126.8(19) } \\
& \mathrm{O}(11)-\mathrm{C}(17)-\mathrm{C}(16) \quad 113.6(15) \\
& \text { C(19)-C(18)-O(11) 100.6(14) } \\
& \text { N(11)-C(19)-C(18) } \quad 110.6(17) \\
& \text { N(11)-C(19)-C(110)\#1 109.5(11) } \\
& \text { C(18)-C(19)-C(110)\#1 108.5(12) } \\
& \text { N(11)-C(19)-C(110) 109.5(11) } \\
& \text { C(18)-C(19)-C(110) 108.5(12) } \\
& \text { C(110)\#1-C(19)-C(110) 110(2) } \\
& \mathrm{C}(22)-\mathrm{C}(21)-\mathrm{C}(26) \quad 117.7(17) \\
& \mathrm{C}(22)-\mathrm{C}(21)-\mathrm{Hg}(2) \quad 124.6(15) \\
& \mathrm{C}(26)-\mathrm{C}(21)-\mathrm{Hg}(2) \quad 117.7(14) \\
& \mathrm{C}(23)-\mathrm{C}(22)-\mathrm{C}(21) \quad 123(2)
\end{aligned}
$$




$\begin{array}{ll}\mathrm{C}(22)-\mathrm{C}(23)-\mathrm{C}(24) & 118.6(19) \\ \mathrm{C}(25)-\mathrm{C}(24)-\mathrm{C}(23) & 120.5(18) \\ \mathrm{C}(24)-\mathrm{C}(25)-\mathrm{C}(26) & 123(2) \\ \mathrm{C}(25)-\mathrm{C}(26)-\mathrm{C}(27) & 124.4(16) \\ \mathrm{C}(25)-\mathrm{C}(26)-\mathrm{C}(21) & 116.7(17) \\ \mathrm{C}(27)-\mathrm{C}(26)-\mathrm{C}(21) & 118.9(14) \\ \mathrm{N}(21)-\mathrm{C}(27)-\mathrm{O}(21) & 117.5(17) \\ \mathrm{N}(21)-\mathrm{C}(27)-\mathrm{C}(26) & 125.9(15) \\ \mathrm{O}(21)-\mathrm{C}(27)-\mathrm{C}(26) & 116.6(15) \\ \mathrm{O}(21)-\mathrm{C}(28)-\mathrm{C}(29) & 103.0(15) \\ \mathrm{N}(21)-\mathrm{C}(29)-\mathrm{C}(210) & 108.7(11) \\ \mathrm{N}(21)-\mathrm{C}(29)-\mathrm{C}(210) \# 2 & 108.7(11) \\ \mathrm{C}(210)-\mathrm{C}(29)-\mathrm{C}(210) \# 2 & 109(2) \\ \mathrm{N}(21)-\mathrm{C}(29)-\mathrm{C}(28) & 103.8(14) \\ \mathrm{C}(210)-\mathrm{C}(29)-\mathrm{C}(28) & 113.2(11) \\ \mathrm{C}(210) \# 2-\mathrm{C}(29)-\mathrm{C}(28) & 113.2(12) \\ & \\ & \\ & \\ & \\ & \\ & \end{array}$

Symmetry transformations used to generate equivalent atoms:

\#1 x,-y-1/2,z \#2 x,-y-3/2,z 
Table 4. Anisotropic displacement parameters $\left(\AA^{2} \times 10^{3}\right)$ for 8 .

The anisotropic displacement factor exponent takes the form: $-2 \pi^{2}\left[\mathrm{~h}^{2} \mathrm{a}^{* 2} \mathrm{U}^{11}+\ldots+2 \mathrm{~h} \mathrm{k} \mathrm{a}^{*}\right.$ $\left.b^{*} U^{12}\right]$

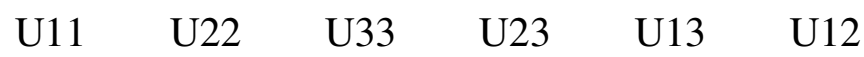

\begin{tabular}{|c|c|c|c|c|c|}
\hline $\operatorname{Hg}(1)$ & $27(1)$ & $55(1)$ & $39(1)$ & 0 & $4(1)$ \\
\hline $\operatorname{Hg}(2)$ & $26(1)$ & $54(1)$ & $40(1)$ & 0 & $8(1)$ \\
\hline $\mathrm{Cl}(1)$ & $34(2)$ & $98(5)$ & $69(4)$ & 0 & $-9(2)$ \\
\hline $\mathrm{Cl}(2)$ & $29(2)$ & $84(4)$ & $83(4)$ & 0 & $24(2)$ \\
\hline $\mathrm{O}(11)$ & $30(6)$ & $89(12)$ & $31(6)$ & 0 & $1(5)$ \\
\hline $\mathrm{O}(21)$ & $34(7)$ & $86(12)$ & $40(7)$ & 0 & $23(6)$ \\
\hline $\mathrm{N}(11)$ & $33(8)$ & $48(10)$ & $53(10)$ & 0 & $12(7)$ \\
\hline $\mathrm{N}(21)$ & $10(5)$ & $60(10)$ & $31(7)$ & 0 & $4(5)$ \\
\hline$C(11)$ & $30(8)$ & $31(8)$ & $24(7)$ & 0 & $12(6)$ \\
\hline$C(12)$ & $17(7)$ & $58(12)$ & $46(10)$ & 0 & $14(7)$ \\
\hline$C(13)$ & $31(9)$ & $65(14)$ & $62(13)$ & 0 & $26(9)$ \\
\hline$C(14)$ & 27(9) & $62(14)$ & $56(12)$ & 0 & $12(8)$ \\
\hline$C(15)$ & $34(9)$ & $71(15)$ & $37(10)$ & 0 & $3(8)$ \\
\hline$C(16)$ & $35(9)$ & $40(10)$ & $34(9)$ & 0 & $-5(7)$ \\
\hline$C(17)$ & 29(9) & 49(12) & $54(11)$ & 0 & $17(8)$ \\
\hline$C(18)$ & $90(17)$ & $51(13)$ & $26(9)$ & 0 & $7(10)$ \\
\hline$C(19)$ & $32(8)$ & $50(11)$ & $35(9)$ & 0 & $15(7)$ \\
\hline
\end{tabular}




$\begin{array}{llllllll}\mathrm{C}(110) & 75(11) & 82(14) & 65(11) & -13(10) & 27(9) & -30(11) \\ \mathrm{C}(21) & 32(8) & 52(12) & 45(10) & 0 & 15(7) & 0 \\ \mathrm{C}(22) & 18(8) & 64(14) & 56(12) & 0 & 0(7) & 0 \\ \mathrm{C}(23) & 46(11) & 72(16) & 33(10) & 0 & -10(9) & 0 \\ \mathrm{C}(24) & 23(8) & 58(13) & 67(14) & 0 & 5(9) & 0 \\ \mathrm{C}(25) & 25(8) & 47(12) & 59(12) & 0 & 18(8) & 0 \\ \mathrm{C}(26) & 16(7) & 40(10) & 42(9) & 0 & 15(6) & 0 \\ \mathrm{C}(27) & 40(9) & 42(10) & 34(9) & 0 & 22(7) & 0 \\ \mathrm{C}(28) & 53(12) & 78(17) & 37(10) & 0 & 13(9) & 0 \\ \mathrm{C}(29) & 28(8) & 54(12) & 25(8) & 0 & -1(6) & 0\end{array}$

Table 5. Hydrogen coordinates ( x 10 $)$ and isotropic displacement parameters $\left(\AA^{2} \times 10^{3}\right)$ for 8.

\begin{tabular}{|c|c|c|c|c|}
\hline \multicolumn{2}{|c|}{$\mathrm{x}$} & $\mathrm{z}$ & \multicolumn{2}{|c|}{$\mathrm{U}(\mathrm{eq})$} \\
\hline $\mathrm{H}(12 \mathrm{~A})$ & 5446 & -2500 & -1936 & $1300(1100)$ \\
\hline $\mathrm{H}(13 \mathrm{~A})$ & 6576 & -2500 & -704 & $10(30)$ \\
\hline $\mathrm{H}(14 \mathrm{~A})$ & 6885 & -2500 & 1924 & 57 \\
\hline $\mathrm{H}(15 \mathrm{~A})$ & 6095 & -2500 & 3636 & $150(150)$ \\
\hline $\mathrm{H}(18 \mathrm{~A})$ & 4306 & -1364 & 5705 & $80(60)$ \\
\hline
\end{tabular}




$\begin{array}{lllll}\mathrm{H}(11 \mathrm{~A}) & 3665 & -5468 & 3769 & 500(300) \\ \mathrm{H}(11 \mathrm{~B}) & 3071 & -4435 & 2640 & 100(70) \\ \mathrm{H}(11 \mathrm{C}) & 3134 & -4272 & 4474 & 80(60) \\ \mathrm{H}(22 \mathrm{~A}) & 9605 & -7500 & 2760 & 56 \\ \mathrm{H}(23 \mathrm{~A}) & 8494 & -7500 & 2879 & 130(140) \\ \mathrm{H}(24 \mathrm{~A}) & 8177 & -7500 & 5334 & 10(30) \\ \mathrm{H}(25 \mathrm{~A}) & 8946 & -7500 & 7532 & 20(40) \\ \mathrm{H}(28 \mathrm{~A}) & 10635 & -8633 & 11260 & 40(40) \\ \mathrm{H}(21 \mathrm{~A}) & 12030 & -5757 & 9488 & 60(40) \\ \mathrm{H}(21 \mathrm{~B}) & 11883 & -5695 & 11206 & 170(120) \\ \mathrm{H}(21 \mathrm{C}) & 11413 & -4585 & 9841 & 80(60)\end{array}$

